\title{
Buone prassi traduttive dei termini del linguaggio medico nelle opere di Galdós: l'esempio di Fortunata y Jacinta
}

\author{
Best Translation Practices of Medical Language Terms in the Works \\ of Galdós: the Example of Fortunata y Jacinta
}

Floriana Di Gesù

Università degli Studi di Palermo, Italia

\begin{abstract}
Riassunto: In quest'articolo, attraverso lo studio di alcuni termini presenti nel romanzo Fortunata y Jacinta di Galdós che si riferiscono all'ambito medico e farmaceutico, si desidera portare avanti una riflessione su alcune "buone prassi" che il traduttore può impiegare nel suo lavoro di riscrittura, considerate come il frutto di un'operazione cognitiva, complesso intrigo di acquisizione, categorizzazione, osservazione riflessiva, elaborazione, sperimentazione e di creazione di dinamiche emotivo-motivazionali che hanno come prodotto ultimo la ricreazione del metatesto. Tali "buone prassi" rientrano a tutti gli effetti negli argomenti da trattare all'interno di una didattica della traduzione dal taglio neurolinguistico.
\end{abstract}

Parole chiave: buone prassi traduttive, categorizzazione, lexicón mental, materia medica, traduzione letteraria.

Abstract: In this paper, through the study of some terms present in the novel Fortunata y Jacinta by Galdós that refer to the medical and pharmaceutical field, we want to carry out a reflection on some "best practices' that the translator can use in his rewriting work, considered as the result of a cognitive operation, a complex intrigue of acquisition, categorization, reflective observation, elaboration, experimentation and creation of emotional-motivational dynamics which has, as its ultimate product, the recreation of the metatext. These 'best practices' fall to all intents and purposes in the topics to be treated within a teaching of translation with a neurolinguistic slant.

Keywords: best practices in translation, categorization, lexicón mental, medicine, literary translation.

\section{L'arte del "dire quasi la stessa cosa" e Galdós}

Lo scopo di quest'articolo risiede nella volontà di offrire al lettore, in ambito di didattica della traduzione, un esempio di alcune "buone prassi traduttive" intese, in questo contesto, come la capacità di acquisizione e applicazione di metodologie specifiche di ricerca, analisi e, soprattutto, categorizzazione che possano permettere al traduttore la realizzazione di un metatesto che soddisfi le aspettative della cultura 
d'arrivo. Tali "buone prassi" si possono configurare come un valido strumento di supporto alla competenza traduttiva.

Nel presente lavoro, quindi, si offriranno degli esempi della metodologia di ricerca ed analisi che il traduttore deve applicare per la resa, nel testo d'arrivo, della terminologia specialistica in ambito medico presente in molte opere di Galdós, che testimoniano il fascino che la materia medica esercitava sull'autore. Tali esempi sono tratti dal romanzo Fortunata y Jacinta e costituiscono, appunto, un campione paradigmatico del lavoro preliminare che il traduttore deve operare.

In questo caso specifico occorre evidenziare che la sfida del traduttore è duplice in quanto egli, non solo è chiamato a confrontarsi con la personalità letteraria di "cotanto autore", ma deve anche dimostrare di possedere le conoscenze tematiche per potere riprodurre, nel metatesto, la materia scientifica presente nel prototesto, consapevole che il linguaggio della medicina, nel corso dei secoli, è stato caratterizzato da un'abbondante produttività terminologica e ciò comporta per il traduttore il misurarsi con il grosso problema dell'equivalenza dei termini da una lingua all'altra.

Nel processo traduttivo, dunque, non sono sufficienti, unicamente, le conoscenze linguistiche ma il traduttore dovrà dimostrare di possedere anche delle approfondite conoscenze extralinguistiche sulla cultura di partenza e d'arrivo, sul contesto storico e socioculturale, sul tema trattato dal testo che si sta traducendo, nonché, nello specifico della traduzione letteraria, dovrà possedere una particolare sensibilità letteraria che si viene a configurare come l'elemento indispensabile per potere trasmettere l'armonia estetica del prototesto.

Di conseguenza, alla ricerca delle fonti documentali seguirà l'analisi linguistica del testo coadiuvata dall'uso delle fonti terminologiche a disposizione, quali dizionarimonolingue e multilingue, specializzati, enciclopedie, glossari, memorie di traduzione e Cat-tools - queste ultime, dal momento che economizzano i tempi di ricerca, rappresentano uno strumento molto efficace. Pertanto, la prassi metodologia che si suggerisce in questo articolo si viene a collocare come un presupponendum traduttivo, per maggiore aiuto e vantaggio nel processo di ricreazione del metatesto, al fine di garantire al lettore una corretta contestualizzazione.

\subsection{II "sapere fare" del traduttore in Galdós}

Entrando nello specifico della traduzione letteraria, l'eclettica personalità artisticoletteraria di Galdós sembra porre a dura prova quel "saper fare" del traduttore, dal momento che la traduzione di molte delle sue opere vede confluire, in un unico prodotto, diverse tipologie traduttive, dalla letteraria alla specialistica e quindi richiede una scommessa da parte del "creatore del metatesto", riassumibile nella considerazione operata da Eco nel 1995 secondo cui:

Tradurre significa interpretare e interpretare vuole dire anche scommettere che il senso che noi riconosciamo in un testo è in qualche modo e senza evidenti contraddizioni co-testuali, il senso di quel testo. II senso che il traduttore deve trovare e tradurre (...) è soltanto il risultato di una congettura interpretativa. II senso non si trova in una no language's land: è il risultato di una scommessa (cit. in Osimo, 2002, p. 60). 
Inoltre, sempre in riferimento a questo "sapere fare" del traduttore che deve dimostrare di sapere gestire tanto la "materia" letteraria quanto quella specialistica, riportiamo un'affermazione di Berman in merito alla differenza traduttiva tra testo letterario e specialistico:
Esprimiamo la differenza tra specialistico e letterario (in senso ampio) dicendo che i testi specialistici appartengono alla categoria di trasmissione di un'informazione determinata, cioè di comunicazione, mentre i testi letterari appartengono alla categoria di trasmissione dell'esperienza umana dell'essere nel mondo (...). (2003, p. 57)

Ma l'opera di traduzione dei testi di don Benito sembra non presentare questi confini così netti dal momento che il traduttore, nel confrontarsi con il prototesto galdosiano, fa esperienza del fatto che i suoi testi appartengono sì alla categoria di trasmissione dell'esperienza umana, per cui li definiamo letterari, ma presentano anche una puntuale attenzione alla trasmissione di una determinata informazione appartenente all'ambito medico e quindi si possono definire, lato sensu, specialistici. Si pensi, per esempio, alle particolareggiate e dotte descrizioni di infermità mentali e fisiche presenti nei testi dell'autore. Potremmo affermare che si assiste, in alcune opere di Galdós, ad un processo di ibridazione testuale che comporta una certa permeabilità delle frontiere tematiche per cui, all'interno del canone letterario, si innesta quello medico grazie, soprattutto, al fascino che tale disciplina esercitava sull'autore e sicuramente al fatto che il periodo in cui egli scrive è denso di cambiamenti e scoperte importanti dal punto di vista della medicina, basti solo pensare a Pasteur, Koch o Lister. Come sostiene Turner in un interessante articolo:
Existe una apasionante relación entre Benito Pérez Galdós y el mundo de la medicina, señalada ya por muchas fuentes, análisis y comentarios; éstos comienzan tratando la ambigua relación entre el doctor Anselmo y el narrador en su primera novela La sombra (1871) para luego dilucidar los componentes de la famosa descripción del manicomio de Leganés en La Desheredada (1881). Después abundan las indagaciones agrupadas en torno a los diversos hechos psíquicos y fisiológicos de toda una gama de locos, cuerdos y epileptoides que encontramos por las novelas contemporáneas. (Turner, 1998, p. 442)

Sempre Turner (1988, p. 446), più avanti, parla della "passione" di don Benito per la materia medica e fa riferimento alla biografia di Galdós scritta da Ortiz Armengol (1995) in cui si fa esplicita menzione alla stretta relazione di amicizia che legava l'autore ad alcuni importanti medici dell'epoca, fra questi: Manuel Tolosa Latour ed il grande Gregorio Marañón. Don Benito in persona ci racconta della stretta amicizia che lo univa al primo:

\footnotetext{
En efecto con Manolo Tolosa Latour, a quién llamábamos familiarmente Dr. Fausto me unía desde tiempo immemorial una amistad cordialísima. Renombrado médico de la niñez curábame también a mí las indisposiclones infantiles que a veces padecía yo. El y su ilustre esposa Elisa Mendoza, que había sido la primera actriz de su tiempo eran los primeros asistentes a mis estrenos... (Memorias de un desmemoriado in Schmidt, 1969, p. 11)
} 
Inoltre, il dottor Tolosa Latour di don Benito afferma:

Tiene afecto hacia la medicina y los médicos, lo prueba su buen gusto de no ridiculizarlos jamás. Celipín el de Marianela será con el tiempo el Dr. Centeno y veremos como estudia antes de llegar a conseguir la borla, Veterinaria y Farmacia; Augusto Miquis, el distinguido médico de la Desheredada es un retrato en cuerpo y alma; las escenas del crup en León Roch y la operación de Teodoro Golfín, el oculista, están estudiadas con exquisito cuidado y descritas de un modo sorprendente; y por fin, entre sus lectores más asiduos y entusiastas, cuenta a no pocos médicos de mucha fama. (Cartas de Manuel Tolosa Latour a Galdós in Schmidt, Ruth 1969, p. 18)

Tolosa Latour, come afferma la studiosa Schmidt (1968: 92), ispirò la figura di Augusto Miquis in La desheredada (1881), in questa figura Galdós riuniva le sue simpatie nei confronti delle nuove teorie degenerazioniste che attribuivano le idee di una predisposizione genetica verso la malattia alle condizioni di povertà e di degrado sociale. Tale influenza è riscontrabile nel testo quando, in merito alla nascita del figlio deforme di Isadora, Miquis afferma: "iMisterios de la herencia fisiológica! Su madre me pregunta si toda aquella gran testa estaría llena de talento. Yo te digo que su delirante ambición y su vicio mental le darán una descendencia de cabezudos raquíticos" (La desheredada 290). In seguito alla "decisiva aparición di La deheredada", come sostiene Fernández (2008) "... comienza a afianzarse una tendencia médica y sociológica en las novelas españolas, íntimamente vinculada a la irradiación de la escuela naturalista".

Stannard (2015) riferisce, in un suo articolo sulla base scientifica del sapere medico galdosiano, che sono più di 50 le figure mediche a cui fa riferimento Galdós nelle sue opere. Lo stesso autore dichiara il suo fascino verso la materia medica in un suo scritto del 1889 in cui afferma: "envidio tanto a los que poseen la ciencia hipocrática, que considero llave del mundo moral" (Pérez Galdós, Niñerías VIII). Stannard, inoltre argomenta che:

Galdos's enthusiasm for medicine was such that combined with his prodigious memory, he packed many descriptive details into his novel well beyond the minimal requirements of the narrative. His love of detail is revealed by the extensive pharmacopeia quoted in Fortunata y Jacinta...It also appears in his clinical representations of the death from tuberculosis of Alejandro Miquis, the mania and delirium tremens of Mauricio la Dura and the congenital syphilis and general paralysis of the insane of Maxi Rubín. For all the broad sweep and vision of his writing, Galdós loved the detail that added to the sense of authenticity that characterizes his narratives and he a-operas driven to include many medical details beyond the knowledge of many laymen. (Stannard, 2011, pp. 332-333)

Da quanto sostenuto fino ad ora, grande valenza sembra assumere il succitato concetto di ibridazione testuale che vede, appunto, il "mundo moral" rappresentato nelle opere dell'autore, fortemente influenzato dalla "ciencia hipocrática", considerata un'importante chiave di lettura per decodificare i mali dell'vomo, prodotto di una deterministica degenerazione.

In quest' articolo, pertanto, attraverso lo studio di alcuni termini presenti nel romanzo Fortunata y Jacinta di Galdós che si riferiscono all'ambito medico e farmaceutico, si desidera operare un processo di analisi che, lungi dall'essere un esame qualitativo 
delle rese linguistiche operate dai traduttori delle opere del genio letterario, vuole avere come fine ultimo l'offrire una riflessione sul lavoro preliminare che deve compiere il traduttore e sulle sue capacità di essere critico davanti al vocabolario tecnico-scientifico ed essere cosciente delle sue limitazioni; di sapere trovare degli equivalenti utilizzando delle risorse terminologiche appropriate e, in ultimo, di sapere trovare degli equivalenti quando non esiste un equivalente.

\section{L'ostinato-destinato rigore del traduttore}

In un altro contesto (Di Gesù, 2019) si è fatto riferimento all'ostinato rigore che caratterizza la labor del traduttore ed in particolare si è fatto accenno al rigore come al metodo acquisito dallo stesso, somma di abilità e di competenze, ovvero di un "saper fare". Proprio nell'atto traduttivo il rigore diventa ostinato, il metodo si fonde con quella operazione cognitiva, complesso intrigo di acquisizione, osservazione riflessiva, elaborazione, sperimentazione e fusione di dinamiche emotivo-motivazionali che porta alla ricreazione del metatesto, di ciò per cui il rigore del traduttore era indirizzato, orientato, insomma destinato.

Nel suo approcciarsi al testo, il traduttore attinge al suo lexicón mental, ovvero a quella mappa mentale che avrà scientemente ordinato secondo categorizzazioni e sottocategorizzazioni che gli permettono di recuperare il dato facilmente. Il processo che si attiva è una complessa operazione di attivazione ed inibizione delle opzioni traduttive. La categorizzazione attivata dal nostro "traslatore" può essere considerata come un processo mentale di organizzazione delle informazioni che si viene a configurare come la base su cui si poggiano tutti i processi di comprensione e produzione linguistica.

Cuenca e Hilferty (1999), riferendosi al processo di categorizzazione lo definiscono come:

(...) un mecanismo de organización de la información obtenida a partir de la aprehensión de la realidad, que es, en sí misma, variada y multiforme. La categorización nos permite simplificar la infinitud de lo real a partir de dos procedimientos elementales de signo contrario o, mejor dicho, complementario: la generalización o abstracción y la discriminación (...) Mediante la categorización agrupamos elementos diferentes en conjuntos, lo cual nos permite pensar, percibir, actuar e incluso hablar. Dicha categorización tiene unas bases comunes a todo el género humano, unos principios generales, pero presenta también algunas diferencias (...) (p. 32)

Parlando in termini neurologici, possiamo affermare che gli elementi che compongono questo database mentale sono organizzati, a loro volta, in grandi reti cognitive interconnesse sia a livello formale che semantico. Sono veri e propri corpora di concetti che portano alla strutturazione di reti semantiche e ciò che viene attivato è un processo di archiviazione attraverso la transizione da una memoria a breve termine (MBT) a una a lungo termine (MLT). L'atto del tradurre è sostanzialmente composto da un'attività di lettura e dalla consultazione di memorie semantiche per la ricerca delle corrispondenze fra le due lingue. Pertanto, il testo da tradurre compie un percorso all'interno della psiche del traduttore per il quale il prototesto, tramite un canale - la lettura - entra nella macchina neurale, ovvero la psiche, che lo elabora fino ad estrapolare il metatesto. II processo che si attiva è quasi immediato, dal momento che, già a partire da una prima lettura che fa il traduttore, si mette in moto 
tutto il suo macchinario neurale. II traduttore, quindi, attinge dal lexicón mental nel quale risiede ed è catalogata tutta una catena semantica di significati e di costruzioni di enunciati a cui corrisponde una rete di circuiti sinaptici (Di Gesù, 2012). A tal proposito López García (2016, pp. 36-37) afferma che "es en el léxico de una lengua donde se comprueba reiteradamente cómo las palabras reflejan determinadas realidades del mundo exterior y cómo a su vez este mismo léxico, al organizarse en campos semánticos, condiciona la naturaleza del referente".

Parlando della neurolinguistica della traduzione López García (2014) sostiene che quando un enunciato viene tradotto in un' altra lingua ciò che a livello neurofisiologico succede è lo stabilirsi di nuove connessioni sinaptiche diverse da quelle dello stadio precedente, ovvero l'analisi della L 1 da sottoporre a traduzione. Nell'atto del tradurre, tali connessioni sono soggette a modifiche ed ulteriori articolazioni: ciò accade quando, per esempio, il traduttore sceglie dei lemmi, quando li mette in relazione in strutture sintattiche che poi adottano un valore semantico-pragmatico. Durante la traduzione, in termini neurolinguistici, il traduttore riceve il testo $A$ da tradurre e lo interpreta dall'enunciato che ascolta fino agli atomi di senso. Ma quando deve intraprenderne la traduzione al testo $B$, non parte da questi atomi di senso, ma da una comprensione globale del testo, per la cui espressione seleziona atomi di senso di $B$, che suppone siano equivalenti a quelli di $A$, associati da connessioni neurali binarie in qualche magazzino della sua memoria, iniziando, quindi, il cammino inverso (López García, 2014). II lavoro del traduttore è, quindi, passare dal caos della comprensione al cosmos della produzione.

Concordiamo con Cimatti quando, in riferimento al lexicón mental, argomenta che: "Ogni parola (...) innesca imprevedibili ed inaspettate risonanze interne alla mente del parlante, in modo tale da influenzarne - anche se spesso inconsapevolmente - il corso dei pensieri" (Cimatti, 1997, p. 131). Il traduttore sa che ha il compito di plasmare un testo che non deve tradire quello di partenza, ma che si deve potere collocare nel sistema linguistico, culturale e letterario della lingua d'arrivo, pertanto deve avere a sua disposizione degli "arnesi da lavoro", degli strumenti che gli permetteranno di operare tale ricreazione per non perdere l'armonia estetica del prototesto.

\subsection{Gli strumenti del traduttore letterario}

In riferimento alla traduzione letteraria Hurtado Albir ben puntualizza che la "sobrecarga estética" rappresenta il differenziale tra le varie tipologie traduttive, infatti, secondo l'autrice:

Las actitudes del traductor general y del literario son diferentes ante los textos que han de traducir, y ello porque los textos literarios se caracterizan por una sobrecarga estética. De hecho, el lenguaje literario podría definirse como todo lenguaje marcado con recursos literarios, es decir, con recursos cuyo objetivo es complacerse en el uso estético de la lengua y en transmitir emociones al lector. Son características propias del lenguaje literario, entre otras: una integración entre forma y contenido mayor de la habitual, y una especial vocación de originalidad. (Hurtado Albir, 2001, p. 63)

Prima di addentrarci nell'elicitazione di quelle "buone prassi" che il traduttore dovrebbe tenere presenti come lavoro preliminare alla sua ricreazione, è importante fare un breve riferimento al concetto di competenza traduttiva che, sempre secondo 
Hurtado Albir, consiste nella "competencia que capacita al traductor para efectuar las operaciones cognitivas necesarias para desarrollar el proceso traductor" (Hurtado Albir, 2001, p. 375). Essa, pertanto, racchiude in sé tutta una serie di sottocompetenze: comunicativa, culturale, tematica, strumentale, psicofisiologica, interpersonale, strategica e comprende le capacità, abilità, conoscenze che il traduttore professionista deve possedere per potere svolgere la sua attività.

In ogni processo traduttivo il traduttore sceglie di impiegare delle strategie che gli possano consentire la riscrittura di cui è l'unico responsabile. La strategia traduttiva si configura come uno strumento di problem solving che implica non solo l'attivazione dei magazzini mnestici e quindi attiva l'innesco del lexicón mental ma, a livello procedurale, richiede anche la scelta di alcuni procedimenti tipici di una traduzione diretta o obliqua (Vinay \& Darbelnet, 1958).

In questo contesto, si è scelto di prendere come riferimento gli studi di stilistica comparata ed in particolare la scelta è ricaduta sul modello di Vinay e Darbelnet (1958) individuato come prototipo di uno dei numerosi e validi modelli di analisi a disposizione del traduttore. II paradigma proposto dalla stilistica comparata si focalizza sul concetto della ricerca dell'equivalenza, asse analitico fondamentale di molti teorici della traduzione, tra cui Nida (1964)².

Per ciò che concerne le strategie di traduzione diretta, essa contempla operazioni quali il calco, il prestito e la traduzione letterale, il secondo tipo include le quattro operazioni di trasposizione, modulazione, equivalenza, adattamento a cui se ne aggiungono altre come la compensazione, amplificazione, generalizzazione, esplicitazione e infine omissione. Ai fini di una didattica della traduzione queste strategie, così come lo studio dei vari modelli di analisi, costituiscono la base metodologica specifica della disciplina traduttiva.

\section{Buone prassi metodologiche}

Prima di intraprendere l'analisi di alcune buone prassi metodologiche, è opportuno sottolineare, nuovamente, che lo scopo di questa riflessione teorica non è quello di analizzare le opzioni traduttive, non si vogliono mettere a confronto le scelte terminologiche dei diversi traduttori dell'opera, dal momento che ciò implicherebbe l'addentrarsi in questioni di stile e di tecnica e questo non è l'obiettivo che si vuole raggiungere in questo articolo. Ciò su cui invece si vuole porre l'accento, all'interno di una didattica della traduzione, sono alcuni passaggi di una possibile fase metodologica preliminare che tiene conto anche degli apporti della neurolinguistica della traduzione, della linguistica cognitiva e della lessicologia (teoria dei prototipi e categorizzazione).

Inoltre, vista l'estensione della produzione del nostro illustre autore, abbiamo preferito, in questo contesto, avvalerci del lavoro di raccolta di dati presente nella tesi dottorale di Vozmediano Hidalgo (2015) il cui titolo è: Ciencias médicas a través de las novelas de D. Benito Perez Galdós, che, a nostro avviso, risulta essere un ottimo

\footnotetext{
I Si vedano tra gli altri anche i lavori di Malblanc (1961), Scavée e Intravaia (1979), Legoux e Valentine (1979), Vázquez Ayora (1977), Newmark (1988).

2 Vedasi il concetto di equivalenza dinamica la cui finalità è ottenere nel lettore del metatesto la stessa reazione che il prototesto innesca sui suoi lettori. Tale equivalenza è centrata sulla corrispondenza comunicativa del messaggio consentendo, a tale scopo, modifiche sintattiche e semantiche nel testo di arrivo.
} 
compendio analitico che ci permette di effettuare una speculazione nei termini sopracitati. L'autrice basa la sua dettagliata analisi sui libri di astronomia, biologia, medicina, igiene e psicologia presenti nel Catalogo ragionato della Biblioteca di Don Benito Pérez Galdós che nel 1951 pubblica il professore Berkowitz dell'Università del Wisconsin ed è oggi conservato al Museo canario di Las Palmas.

Vozmediano Hidalgo afferma:

El estudio de la obra de Don Benito Pérez Galdós desde el punto de vista sanitario y el hallazgo del enorme caudal de conocimientos tanto farmacológicos como patológicos que se traslucen a través de sus obras, nos indujeron a intentar conocer como adquirió Galdós estos conocimientos, encontrando que entre sus mejores amigos estaban médicos de la categoría de don Manuel Tolosa Latour, su hermano Rafael, ambos pediatras y un tercer hermano Ricardo, farmacéutico. (Vozmediano Hidalgo, 2015, p. 30)

In particolare, come già accennato, abbiamo scelto di restringere il campo di ricerca all'analisi di alcuni termini medico-farmaceutici presenti in Fortunata y Jacinta ${ }^{3}$ considerati, appunto, un campione paradigmatico ai fini dell'illustrazione di tali prassi metodologiche.

Pertanto, procedendo nell'analisi di tali campioni, esempio della terminologia medica riscontrata in quest'opera osserviamo che termini come glóbulos, gránulos, pastillas o píldoras non presentano un problema traduttivo, in virtù del fatto che i referenti sono noti all'immaginario collettivo della cultura di arrivo. Termini come papeletas, invece, hanno bisogno di un'attenzione specifica e di una corretta restituzione nella lingua di arrivo, dal momento che una prima ricerca del lemma nei dizionari non contemplerebbe, tra le entrate principali, quella che concerne l'uso in ambito medico di tale termine. La buona pratica che il traduttore deve applicare, a questo punto, è proprio quella di andare a cercare informazioni sui testi del periodo ed un esempio per tutti lo può costituire la Farmacopea Oficial Española del 1884 e la Farmacopea ufficiale italiana del 1892. II traduttore si avvarrà, inoltre, della consultazione dei dizionari specialistici, dei glossari, nonché delle memorie di traduzione in merito all'uso che a quei tempi veniva fatto di queste papeletas, per scoprire che esse avevano la funzione di racchiudere il medicinale che si presentava in forma di polvere affinché non si spargesse, come si riscontra in un passaggio di Fortunata y Jacinta: "lo primero que hizo Maxi fué sacar de un envoltorio de tamano regular, multitud de pequeños paquetes muy bien doblados como los que en la farmacia llaman papeletas". (Fortunata y Jacinta: II, X, 869) o ancora: "...Pues soñé que estaba yo en el laboratorio y que me entretenía en distribuir bromuro potásico en papeletas de un gramo" (Fortunata y Jacinta: I, I, 826). In questa operazione consiste, quindi, quel presupponendum traduttivo a cui si è fatto cenno precedentemente. Il traduttore, pertanto, non deve dimenticare che il suo compito è quello di restituire un testo meta comunicativamente equivalente ed al contempo deve risolvere l'incognita linguistico-culturale e ricercare nella lingua di arrivo quel termine che meglio possa sciogliere la riserva linguistica. Di conseguenza la "buona prassi" che dovrà mettere in moto sarà quindi un'operazione particolare in cui, per

\footnotetext{
3 Per questo articolo si è fatto riferimento all'edizione del libro digitalizzata, dal momento che essa agevolava la ricerca dell'occorrenza dei termini.

https://www.guao.org/sites/default/files/biblioteca/Fortunata\%20y\%20Jacinta.pdf
} 
ritrovare l'equivalente del termine $A$ nel testo $B$ partirà dalla comprensione globale del testo e selezionerà quegli atomi di senso nella sua lingua di arrivo che suppone siano equivalenti a quelli di $A$, comprovando l'assunto secondo cui le parole riflettono determinate realtà del contesto e della circostanza in cui vengono impiegate condizionandone la natura del referente.

Continuando con l'individuazione e la risoluzione delle incognite lessicali altro esempio è fornito dal termine bizma: "también le he hecho una bizma para la cintura que vale cualquier dinero" (Fortunata y Jacinta: IV, VI, 1004); "Acostáronle con no poco trabajo y le llenaron de bizmas" (Fortunata y Jacinta: IV, VI, 563). Anche in questo caso, nel tentativo di risolvere l'incognita linguistica, la procedura che potrebbe applicare il traduttore, ancor prima di ricorrere all'uso dei glossari e delle memorie di traduzione - che potrebbero anche non sciogliere l'incognita linguistica è, appunto, quella della categorizzazione. Ricercando, infatti, la voce lessicale bizma sul Diccionario della Real Academia Española, si troverà la seguente definizione: "Emplasto para confortar compuesto de estopa, aguardiente, incienso, mirra y otros ingredientes". La ricerca della soluzione semantica per questo termine particolare, poco usato, trova un possibile esito proprio nella sua definizione in quanto esso si viene a configurare come un particolare tipo di emplasto. Pertanto, tra le strategie a disposizione per la corretta restituzione nel metatesto si potrebbe scegliere la generalizzazione, usata quando si attesta la mancanza di referenti specifici nel testo di arrivo e quindi optare per la traduzione in italiano del lemma generico emplasto che, oltretutto, appare svariate volte all'interno del testo, un esempio per tutti: "...que se pueda vender en líquido, en píldoras, en pastillas, cápsulas, jarabe, emplasto y en cigarros aspiradores" (Fortunata y Jacinta: IV, I, 823).

Volendo applicare i principi della categorizzazione individueremo l'area concettuale delle preparazioni farmaceutiche ed avremo, pertanto, che il termine emplasto verrebbe a costituire il livello base. L'iperonimo sarebbe il termine cataplasma e termini come il succitato bizma si configurerebbero come iponimi perché costituiscono un particolare tipo di emplasto. In virtù di quanto detto, nella categoria dell'iponimia metteremo anche il termine Diaquilón che era un particolare emplasto de plomo diffusamente utilizzato a quei tempi come preparazione dermatologica: "Mire, allí está esa mujer esperando hace un cuarto de hora... Diez céntimos de Diaquilón" (Fortunata y Jacinta: IV, IV, I, 822). Conoscendo, quindi, l'iponimo Diaquilón e dovendo sciogliere l'incognita linguistica nel testo di arrivo il traduttore potrà optare per la strategia dell' amplificazione aggiungendo al nome del farmaco l'apposizione emplasto de plomo o indirizzarsi verso quella della esplicitazione spiegando che il Diaquilón è una preparazione dermatologica.

Tra i casi di eponimia è interessante fare menzione del seguente: "¿ En dónde están los frascos de Emulsión Scott?" (Fortunata y Jacinta: IV, I, 822). Casi come questo necessitano di un ulteriore approfondimento della fase di documentazione per la risoluzione dell'incognita linguistico-culturale. L'Emulsione Scott è uno sciroppo di olio di fegato di merluzzo prodotto dalla Scott LTD, una delle multinazionali dell'olio di fegato di merluzzo più conosciute tra la fine del Diciannovesimo e l'inizio del Ventesimo secolo, rinomata per avere modificato la ricetta dell'olio di fegato di merluzzo, proverbialmente sgradevole soprattutto per i bambini, aggiungendogli un 
gusto più gradevole come quello del latte 4 . Tale prodotto era distribuito in Italia ed in Spagna con lo stesso nome. II traduttore, una volta recuperato questo importante dato, potrà scegliere come rendere il termine nel metatesto e una prima semplice strategia da adottare è quella di tradurre letteralmente proprio perché il referente culturale è rintracciabile nella cultura d'arrivo. L'altra opzione da considerare è l'operazione dell'esplicitazione dell'eponimo in una frase che potrebbe essere simile alla seguente: emulsione di olio di fegato di merluzzo Scott. In questo caso la "buona prassi" metodologica si concretizza nell'analisi documentale che il traduttore deve compiere al fine di chiarire l'eventuale incognita terminologica.

Un caso interessante è fornito dalla resa nel testo di arrivo del termine hatchisschina presente nelle seguenti frasi: "Le voy a dar la hatchisschina o extracto de cáñamo indiano, que es maravilloso para combatir el abatimiento del Ánimo..." (Fortunata y Jacinta: II, VIII, 861) e "...las píldoras de hatchisschina, que le quieren dar al pobre Maxi, a ver si le levantan y aclaran un poco aquellos espíritus tan entenebrecidos" (Fortunata y Jacinta: III, I, 919). Nella fase della documentazione il traduttore si sarà informato sull'esteso uso di tale sostanza nella farmacopea dell'epoca, ritenuta ottima per la sua azione analgesica molto simile a quella dell'oppio. Anche in questo caso una buona prassi potrebbe consistere nell'attivare un processo mentale di organizzazione delle informazioni, ovvero un processo di categorizzazione analogo a quello precedente in cui l'area concettuale è la stessa, il livello base lo fornisce direttamente il testo, ovvero il cáñamo indiano altresì detto cáñamo índico5, l'iperonimo lo costituisce il termine cannabis e l'iponimo è il termine hatchisschina(hashish) - una sostanza stupefacente psicotropa ricavata dalla cannabis ed in cui il suffisso -ina informa della composizione chimica di quei composti organici e inorganici contenenti azoto, nello specifico si tratta di un azotato insaturo. La ricerca di equivalenti nella lingua meta porterà il traduttore alla consapevolezza di avere a disposizione una strategia appartenente alla traduzione diretta, oppure potrà ricorrere ad alcune di quella obliqua. II ricorrere all'operazione del prestito per colmare tale lacuna linguistica nella cultura d'arrivo si configura come la lectio faciliora. Passando alle strategie di traduzione obliqua, le opzioni percorribili saranno l'omissione o l'adattamento. La prima consiste, semplicemente, nell'omettere il termine, in tale caso specifico l'operazione non comporta scompenso alcuno alla resa nel metatesto, in quanto è il prototesto stesso che fornisce una spiegazione della sostanza. La strategia dell'adattamento, invece, prevede un intervento da parte del traduttore sostituendo il lessema conosciuto nella cultura di partenza con uno più familiare in quella di arrivo. E dal momento che in un passaggio successivo si fa riferimento alle píldoras de hatchisschina, ciò che il traduttore può fare è operare un adattamento per cui, invece di fare riferimento al composto chimico, si potranno nominare le pillole di resina lavorata (hashish).

L'ultimo caso di esempio paradigmatico che desideriamo presentare è costituito dal termine garrotillo presente in più passaggi del libro: "El garrotillo y la escarlatina fueron entresacando aquella mies apretada, y en 1870 no quedaban ya más que nueve" (Fortunata y Jacinta, II, VI, 40); "La más negra era que el garrotillo le cogió al pobrecillo nene tan de filo..." (Fortunata y Jacinta, X, VI, 268). La definizione del termine che riporta il Diccionario della Real Academia è la seguente: "Difteria grave

${ }^{4}$ Cfr. http://www.lifeder.com [30/01/2021].

5 Cfr. https://dle.rae.es/cáñamo [4/02/2021]. 
u otra forma de angina maligna que solía producir la muerte por sofocación"6. La ricerca della resa linguistica nel metatesto spingerà il traduttore ad operare una ricerca documentale che lo porterà a scoprire che tale particolare termine era presente in Spagna già dal XVII secolo, periodo in cui la nazione si vide devastata dall'epidemia del "mal del garrotillo" termine che derivava dal mezzo che allora si usava (la garrota) per strangolare i condannati a morte. II termine era molto diffuso in Spagna come mostra anche il quadro di Francisco Goya, intitolato El Garrotillo, dipinto intorno al 1810, che ritrae un medico che cerca di rimuovere le placche dalla gola di un bambino affetto da difterite. Quasi mezzo secolo prima, nel 1765 fu il medico scozzese Francis Home (1719-1813) a coniare il termine "croup" per designare questa angina soffocante (Semprini, 2020). Quest'ultimo termine per addomesticazione in Spagna diventa "crup" ed è presente anche in altre opere del nostro don Benito, come per esempio, in León Roch in cui si legge: "...agria como un chirrido de un grito en boca de un demonio y parecida a la inflexión del canto de un gallo, de donde viene, según algunos, el nombre de crup (crow)" (León Roch, 855).

Nell'intraprendere la fase della produzione del testo meta ancora una volta il traduttore dovrà organizzarsi un suo processo di categorizzazione ed identificherà il termine garrotillo, a cui nella sua ricerca documentale avrà unito anche quello di crup, come iponimi del livello base che è la difteria ed il cui iperonimo sarà angina maligna. A questo punto per lui si porrà il problema della resa linguistica nella lingua d'arrivo e tra le operazioni di traduzione a lui a disposizione potrà scegliere, in primis, di utilizzare il termine del livello base ed aggiungervi, quindi, l'ampliazione, fornendo indicatori di contenuto per fare comprendere al lettore del metatesto quale sia la zona colpita dalla malattia.

La figura che segue (fig.1) pretende fornire uno schema visivo del processo mentale che dovrebbe attivare il traduttore come complessa operazione di attivazione ed inibizione di opzioni.

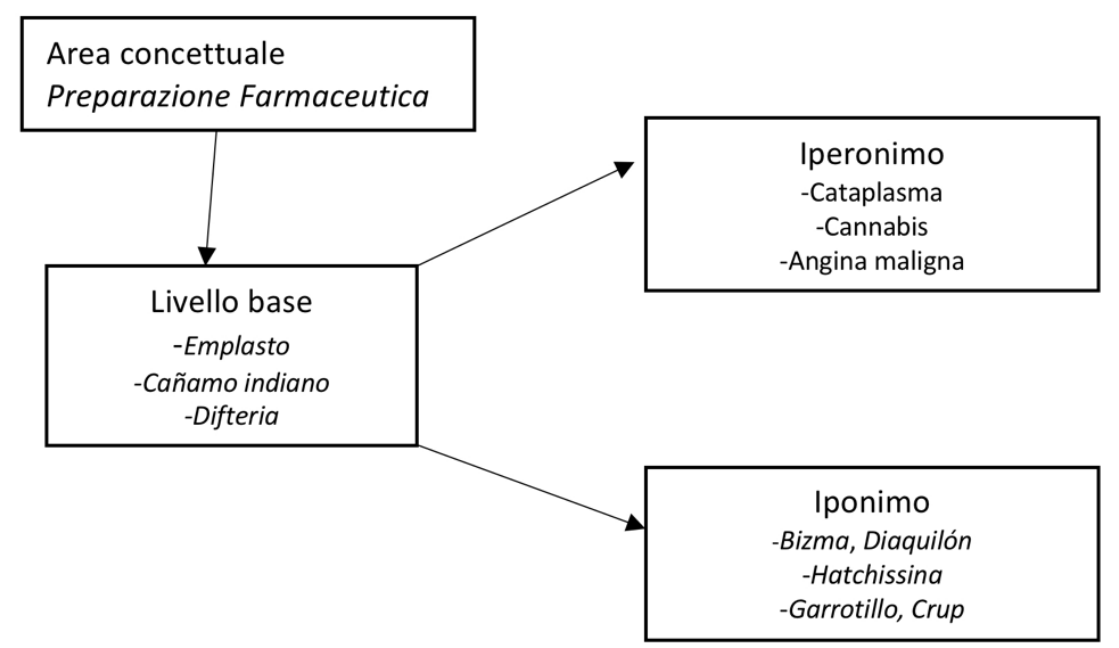

Figura 1. Categorizzazione area concettuale della preparazione farmaceutica

${ }^{6}$ Cfr. https://dle.rae.es/garrotillo [4/02/2021]. 
I succitati esempi sono tesi a dimostrare come il processo di categorizzazione del lessico secondo aree concettuali può essere considerato una buona prassi traduttiva a disposizione del traduttore alla ricerca di un'equivalenza dinamica. Questo processo, infatti, può configurarsi come un tipo di approccio metodologico che il traduttore sceglie di applicare per la sua analisi del testo. Attraverso l'organizzazione del lessico in aree concettuali e la conseguente individuazione di un "livello base" e di iperonimi ed iponimi, il traduttore potrà usufruire di un metodo a corredo dei canonici a lui a disposizione. Questo tipo di approccio mette in rilievo il ruolo "creativo" del traduttore, dal momento che gli permetterà di trovare delle soluzioni efficaci anche in quei casi di difficile recupero del termine dalle fonti terminologiche a disposizione, senza però abbandonare il suo codice deontologico che contempla doveri di probità, dignità, correttezza e competenza che gli impediscono l'alterazione di un testo o la sua interpretazione personale.

Il ricreatore del metatesto deve, quindi, potere avere a sua disposizione una vasta gamma di strumenti metodologicamente affidabili per portare a termine la sua opera.

Nel tentativo di avviarci verso la conclusione della trattazione, possiamo sostenere che tra gli aspetti originali della produzione letteraria del grande don Benito possiamo annoverare la presenza della materia medica. Come afferma Rubín (1970):

Son admirables los cuadros clínicos que aparecen en la obra galdosiana. Los desarrollos de las enfermedades están presentados sin excluir los antecedentes físicos, morales, accidentales y hereditarios de los enfermos, pero con una terminología que no sólo demuestra un aspecto más de la riqueza y elasticidad del lenguaje de Galdós, sino un dominio que desborda la mera brillantez literaria para entrar en el campo de la ciencia. Es evidente que Galdós, en esto, no era sólo literato, sino un gran admirador y aficionado de la medicina. (1970, p. 70)

Al traduttore delle opere in cui è presente questa affascinate commistione tra materia medica e materia letteraria sarà affidato l'arduo compito di sapere restituire nel testo meta ciò che la studiosa Turner (1998) definisce "enredo metafórico" e per riuscire in questa importante impresa dovrà esercitare il suo ostinato rigore, ovvero, dovrà applicare il suo metodo che è frutto della complessa opera di costruzione del suo lexicón mental che, a sua volta, vede nel processo di categorizzazione il suo principio cardine per potere riuscire a "dire quasi la stessa cosa".

Quest'articolo non ha avuto la pretesa di volersi incentrare sulla ricerca di una serie di soluzioni traduttive concrete, proprio perché la loro trattazione per chi scrive non rappresentava il focus argomentativo principale che è, invece, orientato verso una fase immediatamente precedente, ovvero quella della riflessione su alcune possibili "buone prassi" metodologiche "integrate" da strutturare al fine di operare la migliore scelta traduttiva. In questo articolo, infatti, abbiamo voluto proporre i succitati esempi paradigmatici, tratti dal romanzo Fortunata y Jacinta proprio nell'ottica di una promozione di queste "buone prassi integrate" che vedono, per esempio, nel processo di categorizzazione con tutte le sue implicazioni linguistico-cognitive uno strumento da applicare all'analisi traduttologica. Consideriamo di fatto che, per quanto riguarda la restituzione del lessico specifico e non, l'individuazione di aree concettuali e la loro strutturazione in diversi piani prototipici, possano costituire una valida opzione di prassi analitica, in virtù del fatto che viene fornita al traduttore un'impalcatura metodologica su cui basarsi per la risoluzione delle incognite 
traduttive. Inoltre, la metariflessione sui meccanismi neurolinguistici della traduzione, la comprensione del processo neurofisiologico che porta alla organizzazione di un lessico mentale, alla sua strutturazione in grandi reti cognitive interconnesse formalmente e semanticamente (Luque Durán, 2001), costituiscono il terreno su cui costruire la suddetta impalcatura. Quindi, nell'ambito di una didattica della traduzione l'applicazione di "buone prassi" che contemplano l'integrazione di apporti forniti da diverse discipline può essere considerato un ulteriore strumento che possa accrescere la competenza del traduttore.

\section{Bibliografia}

BERGMAN, A. (2003). La traduzione e la lettera o l'albergo nella lontananza. Macerata: Quodlibet.

BerkowItz, H. CH. (1951). La biblioteca de Benito Pérez Galdós. Las Palmas: El Museo Canario.

Cimattı, F. (1997). Linguaggio ed esperienza visiva. Rende: Centro Editoriale e Librario.

CUENCA, M. J. \& HILFERTY, J. (1999). Introducción a la lingüística cognitiva. Barcelona: Ariel.

DI GESÙ, F. (2012). Nella mente del traduttore. La collaborazione tra neurolinguistica e traduttologia. InVerbis. 2012, 1. Milano: Carocci, pp. 37-46.

DI GESÙ, F. (2019). L'ostinato rigore del traduttore. In GARBí, T., Leonardo Da Vinci: Ostinato Rigore. Palermo: UnipaPress.

FERNÁNDEZ, P. (2008). La mujer de letras o la letraherida. Textos y representaciones sobre la mujer escritora en el siglo XIX. Madrid-Toulouse: Servicio de Publicaciones del CSIC.

HURTADO AlBIR, A. (2001). Traducción y traductología: introducción a la traductologia. Madrid: Cátedra.

LÓPEZ GARCíA, Á. (2014). Los mecanismos neuronales del lenguaje. Barcelona: Tirant lo Blanch.

LÓPEZ GARCíA, Á. (2016). Enacción, funciones ejecutivas y léxico. In LÓPEZ GARCíA, Á \& JIMÉNEZ JORQUES, D (ed.), Enacción y Léxico. València: Tirant, pp. 21-53.

LUQUe DURÁN, J. DE D. (2001). Aspectos universales y particulares del léxico de las lenguas del mundo. Granada: Imprendisur.

NIDA, E. (1964). Toward a Science of Translating: with Special Reference to Principles and Procedures Involved in Bible Translating. Leiden: E. J. Brill.

ORTIZ ARMENGOL, P. (1995). Vida de Galdós. Barcelona: Grijalbo.

OsIMO, B. (2002). Manuale del traduttore. Milano: Hoepli.

Pérez Galdós, B. (1966). Memorias de un desmemoriado. In SÁlNZ De Robles, F. C. (ed.) Obras Completas. Madrid: Aguilar, pp. 1655-1659.

RuBín, W. (1970). Medicina en la obra galdosiana. Atlantida, 43, p. 70.

SCHMIDT, R. (1968). Manuel Tolosa Latour: Prototype of Augusto Miquis. Anales Galdosianos 3, pp. 91-94.

SCHMIDT, R. (1969). Cartas entre dos amigos del teatro: Manuel Tolosa Latour y Benito Pérez Galdós. Las Palmas: Ediciones del Excmo. Cabildo Insular de Gran Canaria.

SEMPRINI, A. Storia della difterite. https://www.pediatria.it/storiapediatria/p. asp? nfile=storia_della_difterite [4/02/2021].

StANNARD, W. M. (2011). Galdós and Medicine. Berna: Peter Lang. 
StANNARD, W. M. (2015). Las bases científicas del saber médico de Galdós, Actas del X Congreso International Galdosiano. Las Palmas: Ediciones del Excmo. Cabildo Insular de Gran Canaria, pp. 225-234.

Tolosa LATOUR, M. (1889). Niñerías. Madrid: Tip. De Manuel Ginés Hernández.

TURNER, H. (2000). Creación galdosiana en el marco de la medicina, Actas del XIII Congreso de la Asociación Internacional de Hispanistas, vol. 2. Madrid: Castalia, pp. 441-447.

VINAY, J-P. \& DARBELNET, J. (1995). Comparative Stylistics of French and English. A Methodology for Translation. Amsterdam, Philadelphia: John Benjamins.

Vozmediano Hidalgo, M. L. (2015). Ciencias médicas a través de las novelas de D. Benito Perez Galdós (Tesis doctoral). Madrid: Universidad Complutense. 